\title{
THE IMPORTANCE OF GENDER-AWARE DESIGN IN DIGITAL HEALTH WEARABLES: A CO-DESIGN STUDY FOSTERING SUN PROTECTION BEHAVIOUR IN YOUNG MEN
}

\author{
Khayamian Esfahani, Bahar \\ University of East London
}

\begin{abstract}
The rise in the number of people living with long-term conditions is precipitating a shift towards the development of wearable technologies that can monitor our health remotely. This shift makes it possible to rethink the healthcare structure towards awareness and proactive prevention instead of treatment through empowering users, e.g. patients for self-management of their health. In a sector towards individual specialization and relying on personal healthcare management, design has a key role in user participation and engagement. This paper reports a co-design study that empowered the participants as designers to design human-centred interventions that foster sun protection behaviour in young men. The findings from this study show the following: 1) sun protection concepts to enhance young men's engagement in sun protection behaviour, 2) the integration of gender-aware design in digital health wearables contributes towards better user engagement in wearable technologies of the future.
\end{abstract}

Keywords: User centred design, Participatory design, Design process

\section{Contact:}

Khayamian Esfahani, Bahar

University of East London

Engineering

United Kingdom

b.khayamian@gmail.com 


\section{INTRODUCTION}

The cost-effectiveness of technology-enabled care services (TECS) has become one of the major market growths towards accessible healthcare for patients, carers and health care professionals (NHS, 2018). The current technological innovations and the integration of artificial intelligence (AI) provides opportunities in the development of wearable technologies as a means of facilitating user engagement in healthcare through personal devices (Karen Taylor, 2015). In this context, wearable devices are central to healthcare delivery linking personal devices such as mobile phones and smartwatches to the external environment. This pattern is accelerating as users are becoming empowered and more independent in monitoring their own healthcare and self-management (Thuemmler, 2017). This has led to a significant shift in the role of current global design and technology agencies such as Apple, Google, and Philips towards digital innovation for the development of wearable technologies in healthcare. Also, their partnership with hospitals and pharmaceutical companies has shifted the market growth in the developments of personal health platforms through digital wearables for diagnosis, treatment, patient-self monitoring, and prevention. This shift has influenced the design of wearable technologies towards the technology-push approach, which has led to further challenges for active participation in user engagement with wearable devices (Karen Taylor, 2015). The NHS Institute for innovation and improvement stated, "Despite the strong official commitment to developing a patient-led service, our results suggest the UK is not performing well when it comes to involving patients in their care" (NHS, 2018).

One of the identified challenges within the context of wearable technologies for healthcare is the role of user and their empowerment to take an active role in their healthcare (Karen Taylor, 2015). However, the current digital wearables are often technology-driven without the involvement of the end-user (Karen Taylor, 2015). There is abundant room for further progress in user participation and engagement in their health beyond the current focus on technology end or healthcare provider end. To tackle this, design plays an integral role in the transformation of how healthcare is delivered remotely with increasing effectiveness and efficiency (Cooper, E, Tsekleves, 2017). This makes it possible to rethink the healthcare system towards preventive action instead of treatment.

To illustrate this, the investigation was conducted in the context of fostering young men's engagement in sun protection behaviour. Co-design sessions were deployed to empower the participants as designers to devise a sun protection intervention based on their perspectives for the improvement of sun protection practices in young men. This led to design outcomes aimed at addressing the low levels of sun protection in young men. This article proceeds as follows: Section 2 discusses the low levels of sun protection behaviour in young men and continues with the role of design and technology in wearable technologies. Further, this section reviews the human-centred design approach to achieve the desired health-related behaviour and in the context of sun protection behaviour. Section 3 is concerned with the role of wearable technologies as preventative interventions to enhance risk-taking behaviours. Also, it reviews the existing wearable devices that alert sun protection. Section 4 discusses the deployed co-design sessions to potential design interventions to enhance young men's engagements in sun protection behaviour. Section 5 draws together the key findings, and it opens suggestions for design implications of gender-aware design to enhance user engagement in their own healthcare and self-management.

\section{SKIN CANCER}

Skin cancer is the most preventable cancer and one of fastest-growing cancer in the UK (Cancer Research UK, 2014). Excess sun exposure and sunburn are primary causes of skin cancer and only by protecting the skin from the sun can it be avoided. According to Cancer Research UK, $85 \%$ of skin cancer cases can be prevented through sun protection behaviour. The National Institute for Health and Care Excellence (NICE, 2012) has explained: "sun protection behaviour plays a crucial role in the prevention and the treatment of skin cancer". To reduce the risk of developing skin cancer, attitudinal factors associated with engagement in a range of sun protection behaviours such as using sunscreen and wearing protective clothing is necessary as well as avoiding sunburn through wearing a hat and staying in the shade. According to the Cancer Research UK, sunscreen reduces the risk of developing skin cancer to $40 \%$ through protecting the skin from the sun's UV radiations. However, despite the growth in the market for sun protection products produced by the cosmetics and sunscreen industries, young men age 18 to 24 are more at risk of developing skin cancer due to the low levels of sun protection behaviour (Cancer Research UK, 2014). To tackle this health-related problem that aims to achieve behaviour change, we need to look for another position that results in the desired healthy behaviour. The latest skin 
cancer statistics for the UK show at least 100,000 new cases of skin cancer are diagnosed each year and kills over 2,500 people which is seven people every day. This is an important task as the rates of skin cancer and mortality incidence can be reduced and ultimately prevented in young men through the enhancement of their sun protection behaviour.

\subsection{The role of design and technology}

The recent emerging focus on personalised healthcare optimises the importance of design to enhance the development of healthcare centred on the patients' experience. This has also raised the contribution of the current global design firms such as IDEO to design innovatively personalised and patient-centred healthcare services (IDEO, 2018). An integral role of design in improving wearable technologies for healthcare starts with understanding and influencing the experience of users (e.g. patients) by providing a human-centred experience (Chamberlain and Bowen, 2006).

As discussed in Section 1, the cost-effectiveness of technology-enabled care services (TECS) has become one of the major market growths towards accessible health technologies for patients, carers and health care professionals (NHS, 2018). This has led to a significant shift in the role of current global design and technology agencies such as Apple, Google, and Philips towards digital innovation for the development of innovative and human-centred wearable devices with the support of healthcare providers and patients (Apple, 2018; Google, 2018; Philips, 2018). Their partnership with hospitals and pharmaceutical companies has shifted the market growth in the developments of personal healthmonitoring systems and personal health platforms. Their current technological innovation provides opportunities in the development of personal health monitoring devices (Jeong et al., 2016).

This triangulation between the design agencies, technology providers and the stakeholders in the healthcare system, products and systems drives the current healthcare transformation globally. An example of the resulting technologies includes Google, Apple, and Philips for personal health platforms (Karen Taylor, 2015).

In 2014, Apple launched a health informatics mobile application called 'Health Kit' that enables users to monitor and share their health information such as health conditions, medication, allergies and more. In a similar case, Google has developed personal health monitoring platforms for health and activity tracking. This recent emerging focus on personalised healthcare has optimised the value of humancentred design to enhance the development of healthcare centred on the patients' experience. This has also raised the contribution of the current global design firms such as IDEO to create innovatively personalised and patient-centred healthcare services using the human-centred design approach (IDEO, 2018). Another important aspect of understanding the user/patient experience is related to patient safety rules under the Food and Drug Administration obligation (CDRH, 2017). There is increasing concern over the potential risks for the patients along with the technological advancement in healthcare systems. To minimise the potential risks, the integration of human-centred design principles is required to optimise the user interaction with digital wearables which underpins user engagement towards selfmanagement of healthcare (NHS, 2013).

Human-centred design is a creative approach to problem-solving that prioritises understanding human needs to develop products, services, and systems that are understandable, usable, and desirable for people (Norman, 2013). This approach guides designers to understand human interaction daily through the psychology of human actions that impose its narrative on the field of design of objects. Norman describes the interaction between the user and a physical object through the HCD principles, including 'affordances' and 'signifiers' (Norman, 2013, p.45). Norman defined affordances and signifiers based on the interpretation of how an object is perceivable to define the possible actions for interacting with an object based on the physical characteristics of objects such as size, shape, and colour which act as signifiers to show how users can interact with the objects. He also clarified the concept of affordances and signifiers as perceivable cues related to our interpretation and our past knowledge and experiences applied to our perception.

The implication of HCD principles optimises the human aspect of wearable devices linking people's experience and their motivation to sustain engagement. This is achievable through direct user involvement in designing human-centred interventions in the context. Rinkus et al. (2005) discuss that HCD can be used as the key approach if accompanied by other technological techniques that focus on the advancement of technology. An overview of a research study by MIT Business School in 2014, demonstrates that the majority of new products, systems and services fails not because of technology 
advancement but due to the neglecting of the user need. As illustrated below (Figure 1), technologydriven designs prioritise technology and often neglects the user needs.

Similarly, the market pull strategy is focused on the needs of the user based on existing products and services (Giacomin, 2014). This demonstrates it is important to note that the main challenge to good design remains in uncovering the needs of the user, that is the focus of the HCD approach. For this purpose, HCD benefit this research by understanding the needs of young men in relation to their sun protection behaviour.
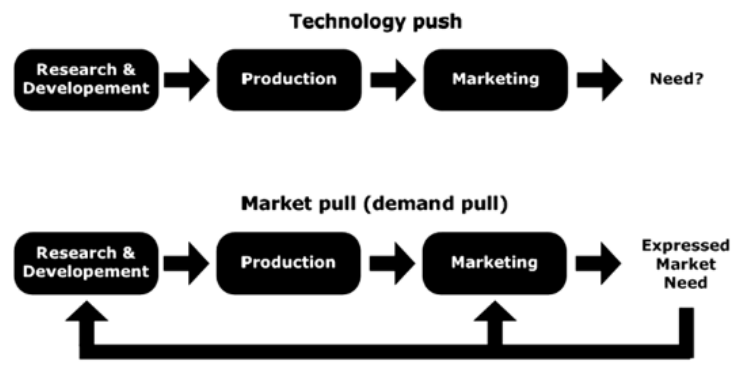

Figure 1. The comparison of technology-push and market-pull business strategies (Giacomin, 2014, p.10)

The current technological advancements in wearable technologies such as EAP (electroactive polymers) stretch sensors has opened a new era for remote healthcare by integrating computer parts such as microelectronic circuits, conductive threads, and supply sensors into garments (Stoppa et al., 2014). The combination of EAP technology through thin and linear actuators is highly suitable for integration in wearable devices for healthcare. The EAP stretch sensor is used for measuring biometric data (technology of measuring and statistically analysing biological data) and body temperature that can be transferred wirelessly to a personal computer.

The developed opportunities for digital wearables contribute towards the development of interventions to enhance the wearability experience and influence on risk-taking behaviours (Stoppa et al., 2014). There are a number of health-related behaviours associated with preventative risk factors such as tobacco use, obesity, and alcohol misuse (Jackson et al., 2012). Desirable changes in health-related behaviours towards prevention lead to the reduction of health incidence rates; therefore, less crowded health care services and subsequent costs. The Department of Health encourages the design of new interventions and programs, particularly in health promotion to stop the annual increase of incidence and mortality rates by changing attitudes and behaviour (Department of Health, 2007). The current policy programs including 'NHS Cancer Plan' and 'Cancer Reform Strategy' by the UK government have focused on areas of primary prevention for cancer-related research to save lives, where design has a key role in health promotion (Department of Health, 2007). One example on areas of primary prevention is skin cancer as the most preventable cancer due to the preventable risk factors associated with avoiding UV exposure (Reichrath, 2014, p.486). The Sun Smart campaign surveyed between 2003 and 2008 to measure trends among sun protection awareness and the effectiveness of the UK campaigns on young men and women age 16 to 24 . The result of this survey demonstrated attitudinal differences in men and women not only in terms of sun protection awareness but also in the way in which the campaigns and studies influence their sun protection behaviour. The results indicated that women are more aware of sun protection practices such as 'avoid sunburn' compared to 2003 and had changed their behaviour. In contrast, the evidence presented from this survey suggests that young men's attitude towards sun protection behaviour from 2003 to 2008 remained unchanged (Cancer Research UK, 2008). Regarding the overall awareness, covering up, using sunscreen, and reducing the time spent in the sun were the least changes in young men's behaviour despite significant changes related to their awareness of the dangers of unprotected sun exposure (Kyle RG, MacMillan I, Forbat L, et al., 2014). The contribution of the current studies and campaigns including 'Sun Smart UK' by the UK Health Department, Cancer Research UK, British Skin Foundation on the health-related behaviours and prevention of skin cancer has driven growth in wearable technologies that promotes sun protection awareness related to the risks associated with sun over-exposure and sunburn (NICE, 2012). 


\subsection{Wearable devices for sun protection}

In 2014, a French company Netatmo, specialising in connected objects, released a sun awareness bracelet, designed by Camille Toupet using technology that measures the level of UV exposure. This wearable device connects to smartphones through Bluetooth to alert the user about the level of their exposure to the sun and alert the user to protect their skin and re-apply sunscreen (Netatmo, 2014). A wearable UV meter wristband called 'SunFriend' consists of a NASA-inspired UV sensor with LED indicators to alert users' maximum sun exposure time compatible to the user's type of the skin (Figure 2). The SunFriend was selected as the best consumer product by NASA aimed to encourage people to get enough vitamin D as well as reducing the rates of skin cancer (Spinoff.Nasa, 2014).

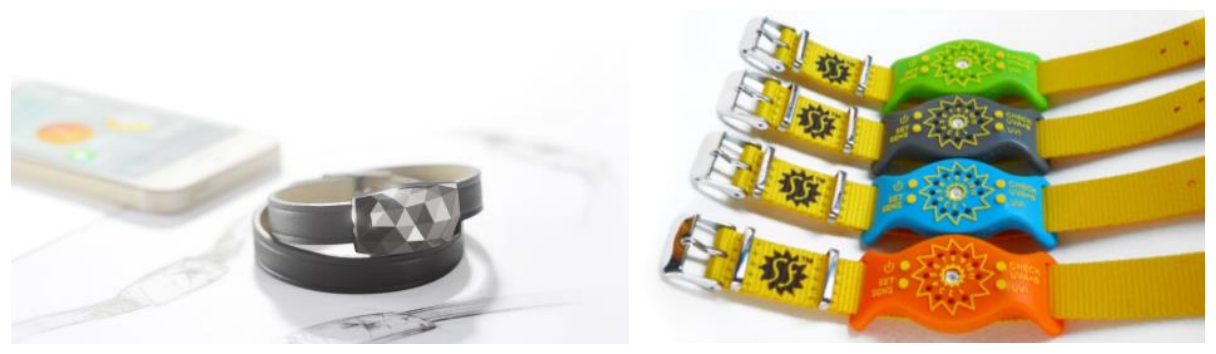

Figure 2. Wearable UV sensors by 'Spinali' Design and 'SunFriend' (Spinoff.Nasa, 2014)

Similarly, a French company called 'Spinali Design' designed swimwear for men and women that communicates levels of sun exposure with smartphones. The embedded UV sensor in the swimwear transfers data to the 'Spinali' mobile phone application to alert the user about sun over-exposure (Spinali Design, 2015). Despite the growth in the design of wearable devices that alerts sun protection, the current studies on exploring men's and women's attitudes towards sun protection behaviour reveal that women are more likely to use sunscreen and seek shade to protect themselves from the sun than men (Courtenay, 2000). Young men are less likely to cover up their upper body parts in the sun than women (Peacey et al., 2006). The current skin cancer incidence for the UK is rising by $7 \%$ between 2014 and 2035 which includes a larger increase for males than for females. Therefore, the further challenge has remained in young men's engagement and participation in their own health in terms of covering up, using sunscreen, and reducing the time spent in the sun.

\subsection{Masculinity}

Gender and masculinity impacts men's lack of engagement in health-related behaviours (Courtenay, 2000). Masculinity is the core theme of understanding young men, defined by Connell as men's endorsement of traditional attitudes and behaviour that is socially constructed (Connell, 1987). Masculinity is a set of attributes associated with enactments of feelings of superiority concerned with dominance into behavioural patterns that indicates toughness and is the opposite of vulnerability (Courtenay, 2000). From this perspective, men's core masculine characteristics are the enactments of being strong, invulnerable, enduring and illness can be perceived as a challenge that indicates weakness or defeat (Mathewson, 2009, p.3).

To understand the concept of masculinity, we need to move on to the core concept of gender. More broadly, to understand gender, we need to determine what is meant by sex and in particular sexuality. The concept of sex is biologically determined and fixed at birth. Sex explains the biological sexual orientation of a person and classifies people based on their natural biological characteristics as male or female. However, expressing sexuality is embedded in our cultures that are in relation to the person's sexual orientation and the way people represent their gender that reflects different characteristics associated with gender roles that form masculinity and femininity. According to Bradley (2013), gender is described as the cultural definitions of masculinity or femininity and the power between men and women that are not stable and fixed, but it develops over time in interaction with cultural and social values (Bradley, 2013, p.3). Therefore, masculinity is socially constructed over time and understanding young men experience requires us to move beyond traditional, stereotypical and pre-identified gendered characteristics.

To gain further understanding of masculinity as a social construct in young men, the following reviews the key theorists with a significant contribution to the field of gender studies regarding the social construction of gender as a 'performance' including the leading gender theorist, Judith Butler. It is 
necessary here to clarify what is meant by gender as a 'performance'. Butler's theory of gender performativity is a principal determining factor that goes beyond normative gender categories established in society.

Gender is a set of performative acts as a lived experience produced through the interplay of acts by subjective experience. The theory of gender performativity destabilises categorising gender performances that produce a particular normative identity being a man or women, but the gender performance is related to the interplay of natural, cultural, and social structure in the society. In line with the debates concerning the notion of gender and performativity by Judith Butler, Frosh et al. describe the construction of gender as not a biological sense or genetically formed but a social construction of actively 'doing gender' with a relational nature. In the same vein, Frosh et al. describe masculinity and femininity as new and subjective replication of actions, gestures and movements that renders one masculine or feminine. Judy Wajcman (1999) demonstrates the ways that technology reflects the gender and its relation to men and masculinity. Wajcman argues the links between technology, gender and society and that technology can be strongly against women and femininity. According to this determinist, the development of technology has been influenced by men's minds and interests that enable social change (MacKenzie \& Wajcman, 1999). Furthermore, the influence of gender inequality and masculinity have grown a men technological worldview that impacts the social shaping of technology. Wajcman fully acknowledges the importance of valuing technological developments based on social values and the society's need rather than masculinity or femininity to conceptualise the current technological developments with equality for both genders.

As discussed in section 2.1, the primary role of applying HCD in design processes is aimed at meeting the needs of people. It ensures that the product is understandable and usable. Although HCD is the most comprehensive account of design principles that explores human needs, this approach is based on a broad understanding of the shared values and common actions of all people as men or women. HCD take account of gender as static and fixed based on the stereotypical norms in terms of how people are perceived as masculine or feminine about their experience. On this basis, instead of repeating the problem, design can address this problem by adding gender awareness to the HCD process that understands how to improve the interaction between a person and a product. HCD provides guidance to explore the elements that fulfil the needs through understanding the underlying meanings people attach while interacting with objects. These methods reveal different levels of knowledge about the user and the contexts. These different levels of knowledge about the context are classified into two categories: 1) explicit and observable, and 2) tacit knowledge (Visser, 2009). Explicit knowledge is the knowledge that is clear and identifiable verbally, and a conscious knowledge that is recognisable through the actions of people based on facts and rules. Conversely, tacit knowledge is subconscious, personal and known to the user, but others can be expressed explicitly as it is linked to skills and experiences.

Furthermore, tacit knowledge is described as "knowledge that people can act upon but cannot readily express in words" (Visser, 2009, p.4). To achieve this, the co-design technique was adopted, where the user involved in the design phase beyond the traditional concepts of design for users to design with users. This study provides an opportunity for potential users to generate design interventions that foster sun protection behaviour in young men.

\section{METHOD}

\subsection{Co-design}

Co-design sessions were deployed beyond the traditional concepts of 'design for users' to 'design with users'. Co-design is a participatory approach with direct user involvement and empowering them as the expert of 'their experiences'. The co-design technique involves the user in the design process to meet their needs from their perception when the user is in full control and empowered in the sessions (Holtzblatt et al., 2005). In this technique, participants are actively involved and expose their deep experience through their designs in relation to the context (Norman \& W. Draper, 1986). It also illustrates participants' tacit knowledge or hidden needs that are not fully known to the user that people can act upon, but cannot express in words (Visser, 2009, p.4).

This study was conducted through eight sessions as the nature of co-design requires a small group of participants for a more focused and in-depth result (Simonsen and Robertson, 2013). A total of eight groups with four to six participants took part in this study for 40 minutes in the form of idea generation to generate new interventions that foster sun protection behaviour in young men. In total, 23 male 
participants and seven female participants participated from University of Brighton, and each session followed the same structure. The purpose of this was to highlight the differences in group dynamics, specifically the participants' behaviour, and the enactment of their gender in relation to these different groups. The different dynamics of male-only groups, as opposed to mixed-gender groups, provide an opportunity to look at the group dynamics in both categories and see how this was linked with the participants' enactment of gender and masculinity.

During the sessions, all participants were encouraged to ideate sun protection interventions to improve young men's sun protection behaviour. The participants started by brainstorming ideas as a group and identifying the specific challenges around their sun protection practices on the beach. The nature of this method empowers the participants to become the experts and design interventions based on their reflections. They immediately began to brainstorm ideas about their sun protection practices, as well as talking about the particular challenges they had faced when using sunscreens.
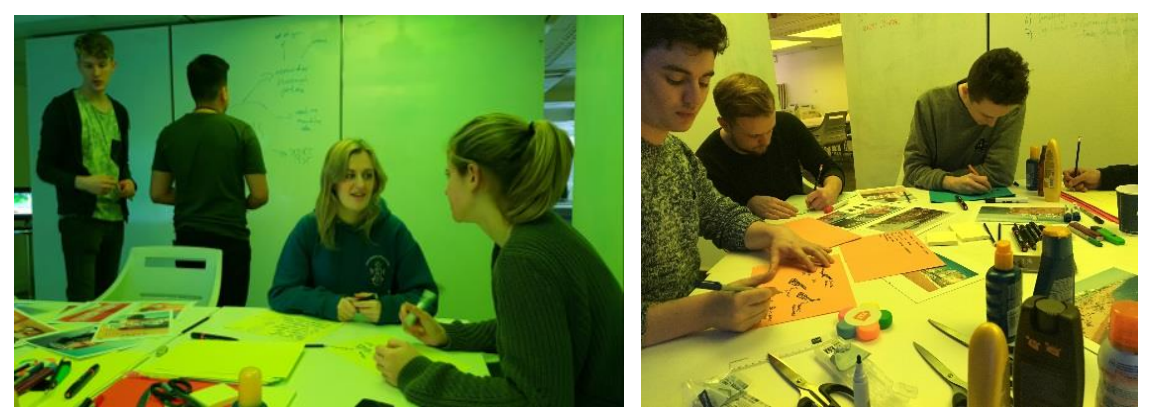

Figure 3. Participants engaged in the co-design session (author's photograph)

\subsection{Results}

The following discusses in detail, as an exemplar of the whole study, four design outcomes that emerged, aimed to improve young men's sun protection behaviour. The following outcomes illustrated below are designed by the male participants (Figure 4). As the participants said: "Our design started with how to remember to apply sunscreen, we designed a digital wearable product that can indicate the UV exposure level by changing colours and reminds people to apply sunscreen. This product would be in forms of a smart wristband, hat, phone case, towel, and goggles". It is clear from the participants' words that their designs are inspired by wearable technology such as smart clothing that detects the sun exposure level to remind the user to apply sunscreen.
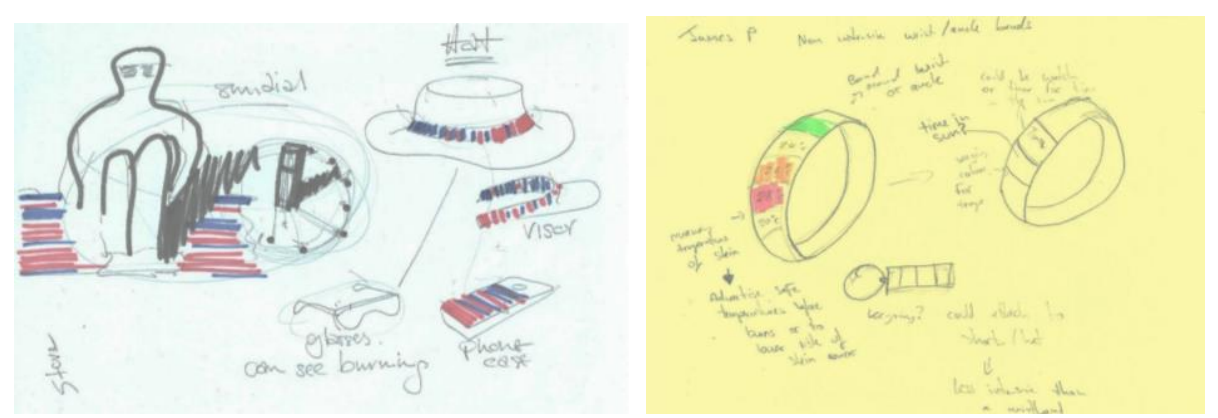

Figure 4. The design outcomes by two male participants (author's photograph)

The Comparisons between the groups were made using analysis of commonalities and differences in relation to their gender awareness. The group dynamic with young men contributes in similar ways of expressing their interests and motives through exploring who's talking the most and who took the lead, who sparking the ideas and who are enacting passive ways of masculinities such as popular forms of being a heterosexual. The enactment of heterosexuality was noticed also the male participants were imitating others in the group and more imitation and following were noticed in the mixed group than the male-only group.

From the HCD perspective, the affordances here are presented as wearables with technological possibilities. The signifier here is the colour coded interface that incorporates technology to remind them of sunscreen application. In particular, the participants said they are more willing to apply sunscreen through this because 'the product will not lie'. At this stage, the participants' indicating their motives 
and reliability towards science and technology. According to Wajcman, technology reflects the ways gender and masculinity in relation to men (Wajcman \& Wheeler, 1985). This is related to the ways gender enactment to construct masculinities in relation to science and technology. We can see the participants performing their gender implicitly, which has influenced the physical appearance of the design outcomes. The characteristics of their design are central for the participants to construct their identity and maintain gender boundaries as a way of expressing their masculinity.

Further, all the male participants indicated that their main worry for sun protection and applying sunscreens is regarding the application of sunscreen on their body. It is understandable from the ways they present themselves towards stereotypical gender boundaries related to the ways heteronormativity shapes their perceptions (Connell, 2005). This indicates the understanding of masculinities related to the participants' expression of heteronormativity through expressing their avoidance of same-sex body contact.

Next, two female participants design the following design outcome. As the participants' said: "this is a female body shape sunscreen bottle and a sponge soaked in sunscreen and will be designed for men and women similar to women's makeup sponge for applying makeup foundation or moisturiser, the colour will be pink or light pink".
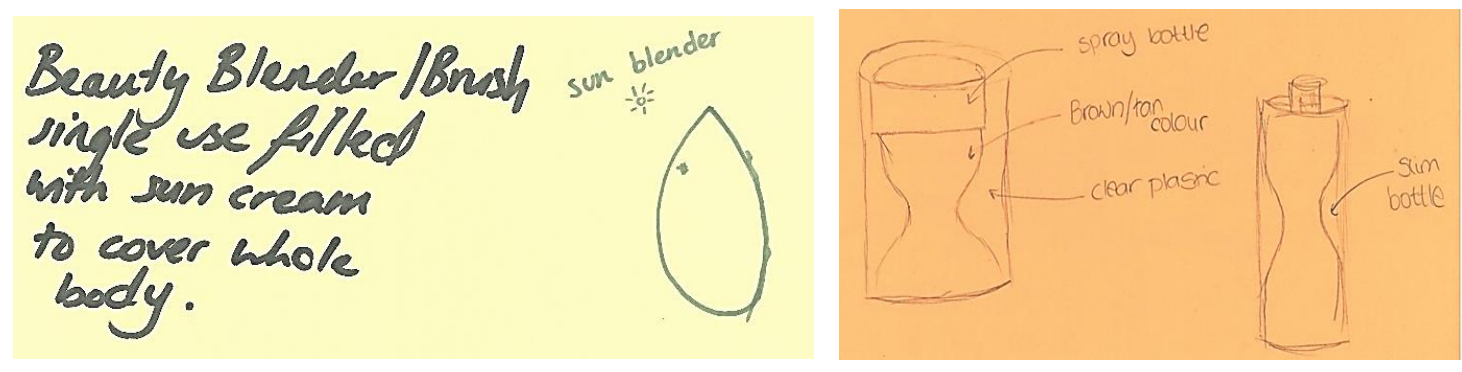

Figure 5. The design outcomes by two female participants (author's photograph)

In their own words, this design outcome is similar to the women's makeup sponge in a bright pink colour. Although all participants were asked to design sun protection interventions for young men, yet here the female participants portrayed their interests and motives influenced by their gender experience with some degree of familiarity. Their design portrays a similar object that is convenient and comfortable for women associated with culturally established and feminine characteristics. As illustrated in Figure 5 , the overall form of this concept is rounded, and an organic shape driven by their experience in the development of this design outcome. Again, the participants' own gender contributed to the physical characteristics and attributes of the affordances and signifiers emerged in their design.

The emerged attributes expressed by the female participants are similar to the particular characteristics of popular products for everyday use targeted at women. This association reveals their motivation identified in the language of affordances and signifiers in a way that is according to their own experience. Up to this point, the comparison of these results pinpoints the significant role of gender in relation to the design outcomes and the participants' own gender perceptions. This evidence suggests the participants' gendered enactments are traced and characterised in the design of affordances and signifiers.

As demonstrated above, the female participants said the appearance of their design is similar to female body shape. Perhaps the characteristics of this design are influenced by the participants' own experience as female participants. For the female participants, rounded and curvy lines are characterised as feminine based on a female body shape highlighted in their design. In this context, the influence of the female participants' own gender perceptions has influenced the overall design which incorporates the design of affordances and signifiers. This can be linked to their previous experiences and the preferred characteristics emerged in the demonstrated design outcome. From the HCD perspective, the overall physical characteristics of the illustrated designs are influenced by the participants' own gender expressed in different ways as the participants seek to conform to the normative and pre-identified gender roles.

From the HCD perspective, the affordances in this design are rounded, and an organic shaped to be held easily, and the spongy characteristic signifies the sunscreen application. These characteristics show similarities with products targeted at women featuring bright colours (preferably pink), soft material, organic, and rounded shape. In contrast, often, men's products are characterised by complex, angular shapes, and dark colours (Moss, 2009). Moss relates the use of aesthetics in products that differs in terms 
of shape, colour and material associated with the perception of culturally established male and female gender roles in the society. Also, the same expectations follow how the product will be used. Examples of such designs are gender-specific shaving razors for men and women that differ in terms of shape, colour and material (Ehrnberger et al., 2012). For example, the bright coloured razors are designed for women through specific characteristics, including soft, rounded, and organic shapes. In this context, colour suggests the use of razors targeted at men or women (Goffman, 1987, p. 27). This shows how the participants' reflections are informed upon expressing their gendered experiences related to their own gender identity provoked by their designs.

As shown, both male and female participants portrayed their perception of masculine and feminine attributes in the design of affordances and signifiers. A possible explanation for these differences is related to the participants' general preference amongst their design with an inclination towards their gender identity. We can see traces of participants' gender enactments and the influence of stereotypical gender characteristics in their designs. The emerging meanings that they attach to the products associated with their gender are consistent with constructing their masculine self. This indicates that gender is very strong and that the HCD elements are influenced by the construction of the male participants' masculinity. As discussed in Section 2.1, affordances and signifiers are fundamentally important in understanding how an object can be used by a particular actor and is the fundamental principle of good design (Norman, 2013). Norman defines affordances and signifiers based on the interpretation of how an object is perceivable to define the possible actions for interacting with an object. $\mathrm{He}$ also clarified the concept of affordances and signifiers as perceivable cues related to our interpretation and our past knowledge and experiences applied to our perception. The affordances and signifiers indicate the importance of understanding the way people interact with physical objects (Giacomin, 2014).

The discussed elements pinpoint the participants' own gender reflections embedded in the design outcomes. Also, their reflections have influenced the design of the affordances and signifiers. This analysis is pushed further through a consideration of the male participants' accounts of their gender and masculinity expressed in their designs. The analysis of the design outcomes outlines a range of characteristics in the participants' design related to stereotypes, expressions of gender and masculinity.

\section{CONCLUSION AND FUTURE WORK}

The results show the role of gender in the HCD and how it can be used to foster sun protection behaviour. Also, sun protection concepts to enhance young men's engagement in sun protection behaviour, 2) the integration of gender-aware design in digital health wearables contributes towards better user engagement. The participants' gender and masculine attributes were linked with the gendered characteristics applied in various features of sun protection interventions. We saw the interplay of gender in the way affordances and signifiers were designed in these concepts.

To foster sun protection behaviour in young men it is important to consider how gender is implied in affordances and signifiers. We can facilitate this by combining HCD principles and practices with an increased gender awareness. Doing this produces design outcomes for sun protection that are more appropriate to young men. Taking this kind of approach will move designers to consider affordances and signifiers in new and innovative ways, and this will have considerable implications in areas beyond sun protection design work. It is therefore important to question how the product will affect the users' behaviour that avoids conflict with the user's gender identity and address their needs. We as designers must become more aware of our responsibility and our power to make a change and avoid the influence of own perceptions, preconceptions, and past experiences. This would avoid the mistakes of the previous design through an understanding of gender as a performance when designing new products. The key outcomes of this research contribute to our understanding in the following directions: the first avenue is based on the development of novel sun protection interventions to improve young men's engagement in sun protection behaviour. The second avenue is based on the integration of gender-aware design in wearable devices contributes towards better user engagement in healthcare. Further development of the sun protection concepts to respond to the current policy programs that cover skin cancer prevention such as 'NHS Cancer Plan' and 'Cancer Reform Strategy' by the UK government (Department of Health, 2007). This should be considered in other areas of primary prevention involved in cancer-related research to save lives, where design has a key role in health promotion. The whole design community should consider the changing role of design to shape technology to achieve better 
user engagement in self-health management. This means moving beyond the technology-driven approach towards digital wearables that are gender-aware to influence on risk-taking behaviours such as tobacco use, obesity, and alcohol misuse. Further research is needed to explore the role of gender and perceived barriers that neglect users' gender identity as it is a crucial element of how digital wearables are established as human centred.

\section{REFERENCES}

Cancer Research UK. (2014), "Skin cancer incidence statistics", available at: http://www.cancerresearchuk.org/health-professional/cancer-statistics/statistics-by-cancer-type/skincancer/incidence (accessed 9 November 2017).

Chamberlain, P.M. and Bowen, S.J. (2006), "Designer's Use of the Artefact in Human- centred Design", Designing Accessible Technology, pp. 67-74.

Connell, R. (1987), Gender and Power:Society, the Person and Sexual Politics, Polity Press, Oxford.

Cooper, E, Tsekleves, E. (2017), Design for Health, available at: http://radar.gsa.ac.uk/5329/1/9781472457424_chapter 17 Macdonald.pdf (accessed 26 November 2018).

Department of Health. (2007), NHS Cancer Reform Strategy 2007, Clinical Oncology (Royal College of Radiologists (Great Britain)), available at: https://doi.org/10.1016/j.clon.2008.01.014.

Ehrnberger, K., Räsänen, M. and IIstedt, S. (2012), "Visualising Gender Norms in Design: Meet the Mega Hurricane Mixer and the Drill Dolphia”, International Journal of Design, Vol. 6 No. 3, p. 17.

Giacomin, J. (2012), "Microsoft Word - What is Human Centred Design - What is Human Centred Design.pdf", No. Krippendorff 1989, available at: http://hcdi.brunel.ac.uk/files/What is Human Centred Design.pdf.

Giacomin, J. (2014), "What is human centred design?”, Design Journal.

Jackson, C.A., Henderson, M., Frank, J.W. and Haw, S.J. (2012), “An overview of prevention of multiple risk behaviour in adolescence and young adulthood", Journal of Public Health, available at: https://doi.org/10.1093/pubmed/fdr113.

Karen Taylor. (2015), The Deloitte Centre for Health Solutions, available at: https://www2.deloitte.com/content/dam/Deloitte/uk/Documents/life-sciences-health-care/deloitte-ukconnected-health.pdf (accessed 20 November 2018).

Moss, G. (2009), Gender, Design and Marketing : How Gender Drives Our Perception of Design and Marketing, Gower.

NHS. (2018), “Technology Enabled Care Services (TECS)”, available at: https://www.england.nhs.uk/tecs/ (accessed 26 November 2020).

NICE. (2012), "National Skin Cancer Campaigns", available at: https://www.nice.org.uk/guidance/ph32/documents/expert-paper-2-national-campaigns-uk-and-worldwide-2 (accessed 16 July 2018).

Norman, D.A. (2013), The Design of Everyday Things, Human Factors and Ergonomics in Manufacturing, Vol. 16, available at: https://doi.org/10.1002/hfm.20127.

Peacey, V., Steptoe, A., Sanderman, R. and Wardle, J. (2006), "Ten-year changes in sun protection behaviors and beliefs of young adults in 13 European countries", Preventive Medicine, Academic Press, Vol. 43 No. 6, pp. 460-465.

Reichrath, J. (2014), Sunlight, Vitamin D and Skin Cancer, Springer New York, New York, NY, available at:https://doi.org/10.1007/978-1-4939-0437-2.

Simonsen, J. and Robertson, T. (2013), Routledge International Handbook of Participatory Design, Routledge.

Spinoff.Nasa. (2014), “Activity Monitors Help Users Get Optimum Sun Exposure”.

Thuemmler, C. (2017), "The case for health 4.0”, Health 4.0: How Virtualization and Big Data Are Revolutionizing Healthcare, Springer International Publishing, pp. 1-22.

Visser, F.S. (2009), Bringing the Everyday Life of People into Design, Fac Industrial Design Dep Design Conceptualization and Communication, Vol. Ph.D., available at: https://doi.org/Isbn 978-90-9024244-6.

Wheeler, E. (1985), "The Social Shaping of Technology edited by Donald MacKenzie and Judy Wajcman (Open University Press, Milton Keynesand Philadelphia, 1985) pp. vii + 237, ISBN 0-335-15027-6", Prometheus, Taylor \& Francis Group, Vol. 3 No. 2, pp. 466-469. 